Robert Alexander, Re-writing the French Revolutionary Tradition. Liberal Opposition and the Fall of the Bourbon Monarchy, Cambridge, Cambridge University Press, 2003, 385 p. ISBN : 052180122 2. 109,7 euros.

Raymond Huard

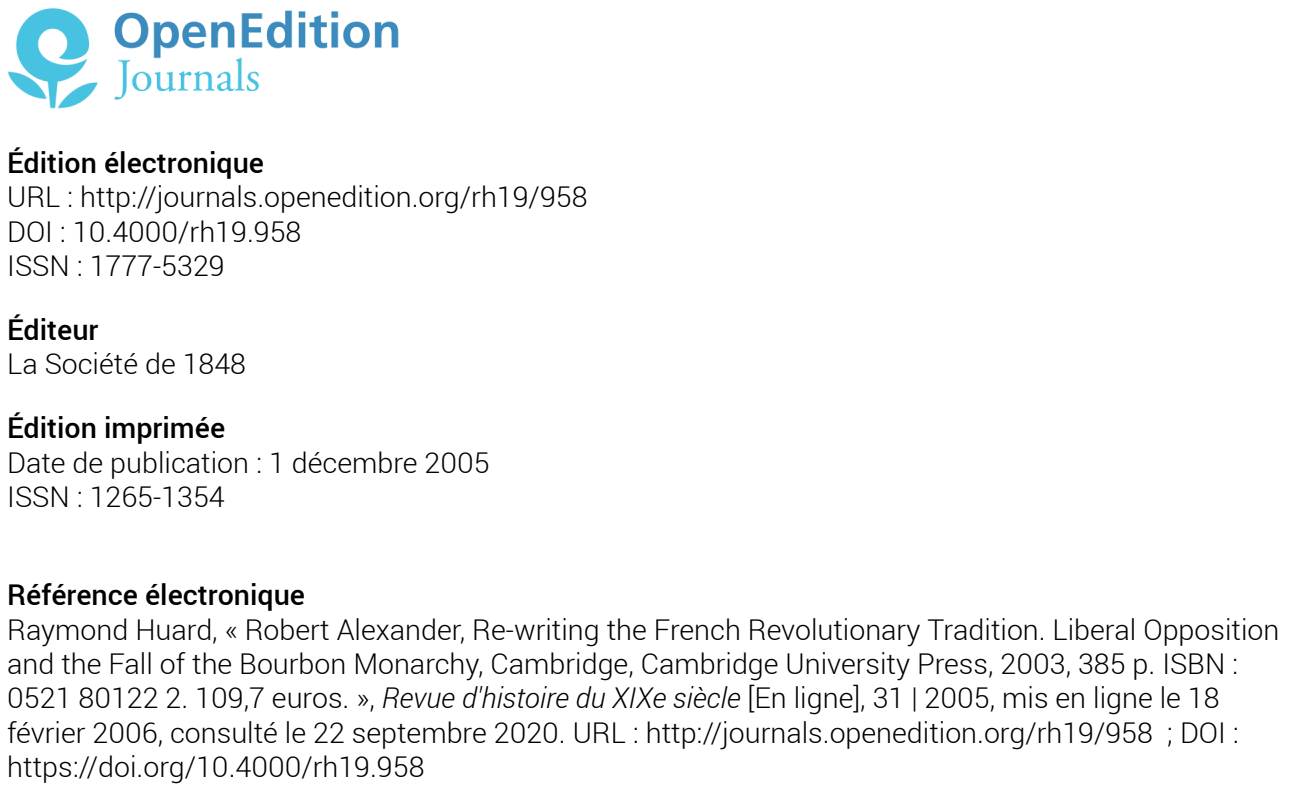

Ce document a été généré automatiquement le 22 septembre 2020.

Tous droits réservés 


\title{
Robert Alexander, Re-writing the French Revolutionary Tradition. Liberal Opposition and the Fall of the Bourbon Monarchy, Cambridge, Cambridge University Press, 2003, 385 p. ISBN : 052180122 2. 109,7 euros.
}

\author{
Raymond Huard
}

Robert Alexander, professeur à l'Université de Victoria au Canada, qui avait déjà publié en 1991 un ouvrage très neuf, Bonapartism and Revolutionary Tradition in France, s'intéresse désormais à la formation et au développement de l'opposition libérale depuis la première Restauration jusqu'aux lendemains de la Révolution de juillet 1830 . Son objectif est de comprendre les raisons profondes du succès de l'opposition libérale en 1830, en se référant à la fois à la nature de cette opposition, à sa tactique et à son enracinement dans la population. Pour cela, sans négliger l'histoire globale au niveau national, il a choisi d'étudier minutieusement quelques territoires particuliers, quatre départements - la Seine-Inférieure, la Haute-Garonne, l'Isère et le Bas-Rhin - qui ne prétendent certes pas épuiser la réalité française, mais qui sont représentatifs de zones géographiques différentes et qui abritent une opposition libérale de force inégale (puissante en Bas-Rhin et en Isère, de force moyenne en Seine-Inférieure, plutôt faible en Haute-Garonne). L'auteur, qui refuse à juste titre de se contenter des sources littéraires, utilise naturellement les études antérieures assez bien fournies dans ces départements et les a complétées par des recherches d'archives et des lectures étendues de sources originales, comme celle du journal La France méridionale de Toulouse. Dans l'ensemble, son information est d'une précision et d'une ampleur tout à fait remarquables. Après une présentation méthodologique, l'ouvrage suit un plan 
chronologique assez classique, en six parties: 1814-septembre 1816, 1816-1820, 1820-1824 1824-1828, 1828-1830, et enfin les lendemains de la révolution de Juillet. Dans chaque partie, il présente d'abord rapidement l'évolution générale de la période (n'oublions pas que le livre est destiné aux lecteurs anglo-saxons), puis se consacre de façon minutieuse aux activités de l'opposition libérale dans les départements indiqués, avec au besoin des comparaisons plus vastes, avec la Côte d'Or, la Sarthe etc... Cela donne un livre très bien construit et surtout très vivant grâce à ces études locales passionnantes. On redécouvre l'action de Villèle à Toulouse et la force du courant ultra dans cette ville, les initiatives très hardies des Grenoblois (l'affaire Didier en 1816, puis l'élection de Grégoire en 1819) et la politique savante des Perier, la combativité exceptionnelle des libéraux alsaciens - les Lichtenberger, Coulmann et autres -, ainsi que les liens étroits que les militants provinciaux (Goyet dans la Sarthe) entretiennent avec les leaders nationaux, Benjamin Constant, La Fayette, élus attentifs à garder un contact étroit avec la base. L'auteur ne néglige ni le rôle des autorités et leur politique "despotique " (terme peut-être un peu fort), ni les tractations des hommes de gouvernement avec l'opposition modérée, ni les alliances éventuelles entre les oppositions de droite et de gauche. Ce qui l'intéresse avant tout, c'est la politique légale, les élections, (qui ne concernent pas seulement, comme il le montre bien, le cercle étroit des électeurs), la presse, les sociétés, les pétitions. En revanche, Robert Alexander attache moins d'importance aux mouvement révolutionnaires (la Charbonnerie notamment et plus tard les tentatives de 1834) longtemps privilégiés par les historiens français.

2 Au-delà de cet apport documentaire où même un lecteur familier de la Restauration trouvera à glaner des faits originaux, deux thèses sous-tendent tout l'ouvrage. La première, c'est qu'il n'y a plus d'avenir, à partir de la Restauration, pour une politique révolutionnaire animée par une minorité active et fondant ses espoirs dans un coup de force. Celle-ci, en inquiétant l'opinion modérée, fait le jeu des forces conservatrices, facilite le raidissement du pouvoir. Pour qu'une stratégie soit opératoire, il faut qu'elle repose sur un large consensus de l'opinion, que l'action légale et le mouvement d'opinion populaire soient en phase. Robert Alexander explique ainsi les succès de 1819 et 1830. Il y a donc sous la Restauration une lutte d'idées pour l'interprétation de la tradition révolutionnaire, car au sein de celle-ci, le courant radical et insurrectionnel est loin d'avoir disparu. Un clivage du même ordre divise les royalistes entre les partisans du coup de force et ceux du compromis avec les idées nouvelles. On peut se demander si cet effort de rationalisation n'est pas poussé un peu trop loin, et si, précisément parce qu'il est fondé sur des cas provinciaux, il fait assez de place à la situation particulière de Paris dans la politique française à cette époque, car c'est tout de même une insurrection parisienne et populaire qui a renversé le régime des Bourbons, comme plus tard celui de Louis-Philippe. Même appuyés par l'opinion, à eux seuls, Thiers, Guizot et La Fayette auraient-ils pu l'emporter en 1830 ?

3 La deuxième thèse concerne le caractère de l'opposition libérale. Certes, ce n'est pas un «parti»: elle est hétérogène idéologiquement - même si ses composantes républicaine, bonapartiste ou orléaniste sont encore peu différenciées -, interclassiste, peu disciplinée, toujours prête à se fragmenter. Sa lutte est menée principalement contre l'État et non contre les autres partis. Néanmoins, aux yeux de l'auteur, elle constitue un pas en avant vers la formation d'un parti, et un modèle qui resservira dans d'autres circonstances (au début de la Troisième République par exemple). On peut 
adhérer à cette vision. En abordant les lendemains de la Révolution de Juillet, l'auteur montre que le débat à propos de la tradition révolutionnaire continue, avec les mêmes aléas. Posons la question : la recomposition politique qui a suivi la Révolution de Juillet, avec l'échec du Mouvement et l'arrivée au pouvoir du parti de la Résistance, n'introduit-elle pas au sein de la tradition révolutionnaire de nouveaux clivages qui modifient le rapport de force entre légalistes et insurrectionnels? 